\title{
A Survey on Community Detection Methods in Social Networks
}

\author{
Mehjabin Khatoon ${ }^{a}$, W. Aisha Banu ${ }^{b}$ \\ ${ }^{a}$ Ph.d(full time),Computer Science \& Engg., B. S. Abdur Rahman University, Chennai-48,India \\ ${ }^{b}$ Professor, Computer Science \& Engg., B. S. Abdur Rahman University, Chennai-48,India
}

\begin{abstract}
A social network is a social structure made up of a set nodes, which represents social actors (such as people, organizations), and edges or lines represents relationship between these nodes or actors. Social networks have important roles in the dispersal of information and innovation, the analysis of such networks, attracted much attention in the research area. The analysis of social network can be done as a whole, which means the representations of all of its actors and identification of structures, present in that social network, that lead to the presence of communities. In the method of community detection, the main aim is to partition the network into dense regions of the graph, and those dense regions typically correspond to entities which are closely related, and can hence be said to belong to a community. In any complex network, communities are able to exchange and offer information because members in one community have similar tastes and desires. The determination of such communities is useful in the context of a variety of applications in social-network analysis, including customer segmentation, recommendations, link inference, and vertex labeling and influence analysis. This paper presents a survey on community detection approaches, which have already been proposed, and also discussing the type of social networks on which those proposed approaches are applicable. This survey can play a significant role in the analysis and evaluation of community detection approaches in different application domains.
\end{abstract}

Index Terms: Community detection, social networks, actors, community structure.

(C) 2015 Published by MECS Publisher. Selection and/or peer review under responsibility of the Research Association of Modern Education and Computer Science.

\section{Introduction}

With the invention of the web 2.0, the users of internet have not only become the consumers of information but also the producers of information. The users of world of web interact with each other, participate in online discussions, exchange different types of information and their views, they form social networks. The interaction among the users in the social networks can be characterized according on the basis

* Corresponding author. Tel: +919941949926, +919790838881

E-mail address: mehjabinkhatoon@gmail.com, aisha@bsauniv.ac.in 
of positions, behaviors or their virtual identities [1]. Social networking has become an extremely important application in the world of internet in the past few years, because of its capability to enable social contact over the internet for geographically dispersed users. A social network can be represented as a graph, in which nodes represent users (e.g. peoples, organizations, groups etc.) and links or edges represent the connections between the users.

Social networks depict the interactions between individuals or entities and are represented by a graph of interconnected nodes. One of the important features of social networks is community structure, and the detection of communities in the area of social networking is an important problem. Till now, many approaches have been proposed to detect the hidden communities in a social network. So there is a high importance for discovering communities, for understanding the type's social networks and detecting the useful and hidden patterns in the aforementioned network [2].

In social networks, the term community has no unique definition till today which can be widely accepted. In Fig. 1, there are three communities in which all the nodes within a community are densely inter-connected with each other and have sparse inter-connection with the nodes belonging to another community. In a social network community, nodes are connected with each other based on their human relationship like friendship, colleague etc. [3].

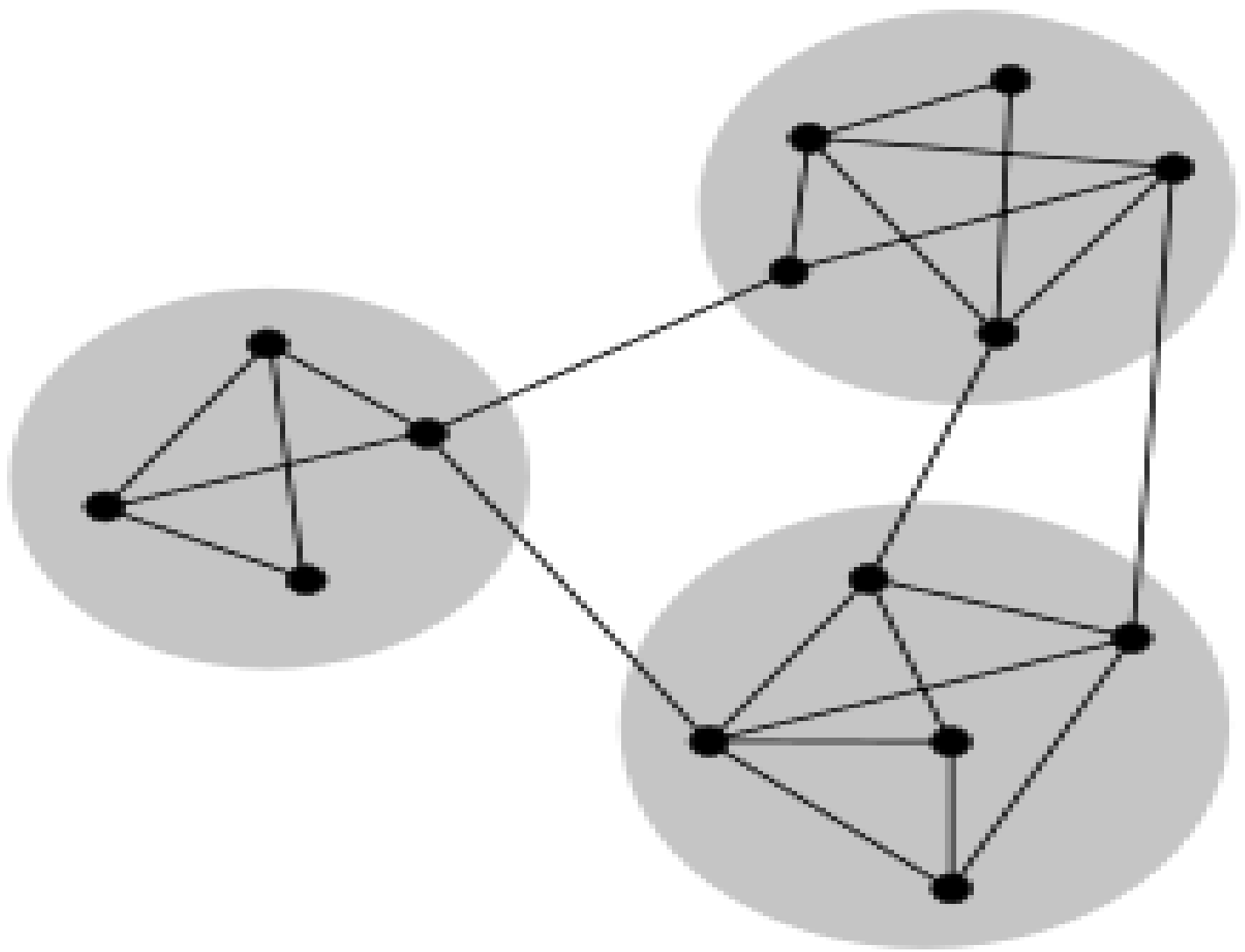

Fig.1. Visualization of community structure with three groups of nodes with dense internal connections and sparser connections between groups. 
The developments of community detection algorithms till now have been done for networks consisting of only few edges. There are many other complex networks which consist of billions or trillions of edges and nodes, for example the online social networks like twitter, facebook having millions of user, and the number is increasing day by day. Many community detection algorithms innovation was done with respect to scalability and many were innovated with respect to static networks. Shortcomings of network on the respect of size were removed by developing algorithm that will work in near-linear time [4].

\section{Related Works}

Many methodologies have been proposed to detect communities in social networks. The proposed methodologies use different data mining algorithms, graph mining algorithms to achieve the task of detection of communities. Few proposed techniques are like, detection of community and sub-community detection in web scale network [3], detection for distributed environment in web scale network [4], detection in integrated internet of things and social network architecture [7], and detection in weighted networks [8].

Newman-Girvan algorithm was proposed for the detection of community and sub-community detection in social networks. It includes two definitive features: first, they involve iterative removal of edges from the network to split it into communities, the edges removed being identified using one of a number of possible "betweenness" measures, and second, these measures are, crucially, recalculated after each removal. The proposed method was implemented on real world networks like Zachary Karate Club, College Football Network and Bottlenose Dolphin Network, and it detects sub-communities in real world networks. The concept of sub-community detection which has two or more than two nodes and the nodes contained in a subcommunity are intra dense connected [3].

For community detection in distributed web scale network, the author's proposed a procedure to find community partitions of networks with billions of edges. The proposed method is based on an ensemble learning scheme for community detection that provides a way to identify high quality partitions from an ensemble of partitions with lower quality [4].

Community detection method in an integrated internet of things and social network architecture uses a graph mining approach in which the formation of community is consider only if the occurrences of two nodes are at most one hop apart and has at least two mutual friends. This approach takes mutual friends as a metric for suggesting friends, and the suggestions for friends would be generated based on the number of mutual friends. The persons connected to the internet are viewed as to be replaced by persons connected to the internet by number of things. So, in the Internet of Things (IoT) there will be more things connected to the internet than the people [7].

Community detection in weighted networks uses the clustering methodology, and its main objective is to maximize total weight of all selected clusters and minimize the similarity between the selected clusters. The total weight of all selected clusters is calculated and also the similarity in between the clusters. In this approach the set of constraints are that every data object is assigned to exactly one cluster, and it assures that every cluster has at least one object. It also ensures that only certain numbers of clusters are selected, and it guarantees that a cluster must be selected if there is any data object assigned to it [8].

There are many other approaches for detecting communities like detection using Dbscan algorithm, detection using Bayesian and expectation maximization technique, detection of overlapping communities which have been discussed in later sections, with its methodology, pros and cons.

\section{Detection of Communities}

In social networks, detection of communities is done by seeing the nodes which are similar to each other, i.e. keeping those nodes in one community. There is more number of nodes inside a single community, than in between two communities. When the nodes of a network can be arranged to form a group such that the nodes are said to have connected internally, then that network is said to have a community structure. 
Community structures are quite common in real networks. Social networks include community groups (the origin of the term, in fact) based on common location, interests, occupation, etc. Metabolic networks have communities based on functional groupings. Citation networks form communities by research topic. Being able to identify these sub-structures within a network can provide insight into how network function and topology affect each other. Such insight can be useful in improving some algorithms on graphs such as spectral clustering.

The identification of community, in a graph of any social network is technically possible by finding node cluster in graph. Clustering in a network can be visualized as the strength-tie inside the community. The research in community detection is to characterize the strong social groups based on social network properties. The properties of social networks can be categorized by static properties and dynamic properties. The static properties are described by the structure of snapshots of graphs in the particular network; while dynamic properties are described by the network structure evolves over time. The above mentioned properties of social networks, may be for un-weighted or weighted graphs, where weights may represent multi-edges (e.g. multiple phone calls from one person to another), or edge weights (e.g. monetary amounts between a donor and a recipient in a political donation network) [5]. Definition of community detection given by different authors is different, and definitions are subjective. Each and every method for detecting communities has different approaches. Thus the result for detecting the communities by different method is different for the same network. So far, the researches done on community detection are in various ways, and are based on an idea of different range. Still in the field of community detection there are so many challenges which are yet to be covered, like the scalability issues [6].

The main advantage in the field of detecting communities is accessing the information from diverse sources and clusters. Detection of communities make possible to exchange and offer information because members in a community often have similar tastes and desires. A community helps to understand the structure of social networks because communities are considered as components of social networks and specify the features as well as functions of the network. Communities' help in the visualization of large-scale social networks, and the relation of communities, clear the process of information sharing and dissemination of information Community detection also gives a vision on network future growth.

\section{The proposed approaches for community detection}

The detection of communities in a network is a complex problem. The evaluation of each method's turns out to be different, and comparing each method's performance proves to be a real challenge. Community detection methods have also been studied in the context of many graph-theoretic clustering algorithms.

A community in a simple language we can say it as a group of nodes which are densely connected by edges. For example, many node clustering algorithms for graphs with the use of shingling techniques, matrix coclustering techniques, and tile determination in matrices can be used for community detection in graphs.

\subsection{Community Detection methods in an Integrated Internet of Things and Social Network Architecture.}

A community detection scheme in an integrated Internet of Things (IoT) and Social Network (SN) architecture has been proposed. The persons connected to the internet are viewed as to be replaced by persons connected to the internet by number of things. So, in the Internet of Things (IoT) there will be more things connected to the internet than the people. The integrated proposed method uses a graph mining approach in which the formation of community is consider only if the occurrences of two nodes are at most one hop apart and has at least two mutual friends. This approach takes mutual friends as a metric for suggesting friends, and the suggestions for friends would be generated based on the number of mutual friends. The problem in complex network of IoT and SN is solved by the graph mining approach. In this proposed approach the smallest community, is a sub-graph with a cycle of length four, and a node can be part of multiple communities, which 
works well for weighted graphs. The results for community detection approach in an integrated environment are more relevant for intra-community methods than inter-community methods [7].

\subsection{Community and Sub-Community Detection methods in Social Networks.}

A new approach was focused for the detection of communities as well as sub-communities occurring in a social network by applying Newman-Girvan algorithm. The implementation was done on real world networks like Zachary Karate Club, College Football Network and Bottlenose Dolphin Network, and it detects subcommunities in real world networks. The concept of sub-community detection which has two or more than two nodes and the nodes contained in a sub-community are intra dense connected. In 2003, Newman-Girvan algorithm was proposed which includes two definitive features: first, they involve iterative removal of edges from the network to split it into communities, the edges removed being identified using one of a number of possible "betweenness" measures, and second, these measures are, crucially, recalculated after each removal. A measure was proposed for the strength of the community structure found by Newman-Girvan algorithm, which gives an objective metric for choosing the number of communities into which a network should be divided. Newman-Girvan algorithms are highly effective at discovering community structure in both computergenerated and real-world network data, and they can be used to shed light on the complex structure of networked systems [3].

\subsection{Community detection methods in weighted network}

A new method was proposed for identifying the communities in a social network through clustering and its main objective is to maximize total weight of all selected clusters and minimize the similarity between the selected clusters. The total weight of all selected clusters is calculated and also the similarity in between the clusters. In this approach the set of constraints are that every data object is assigned to exactly one cluster, and it assures that every cluster has at least one object. It also ensures that only certain numbers of clusters are selected, and it guarantees that a cluster must be selected if there is any data object assigned to it [8].

\subsection{Community detection methods for distributed environment, in Web-Scale Networks}

A distributed community detection methods was proposed for web -scale networks. This approach shows a procedure to find community partitions of networks with billions of edges. The proposed method is based on an ensemble learning scheme for community detection that provides a way to identify high quality partitions from an ensemble of partitions with lower quality. The pre-processing procedure presented for this approach, for community detection algorithms, significantly decreases the problem size. Many algorithms, developed for community detection methods are only non-distributed, in-memory and single threaded algorithms. Algorithms developed, which are very fast and scalable, required to store the complete graph in main memory, so for processing of graphs with billions of edges would require hardware at least hundred GB of RAM. So for overcoming this algorithm, a distributed core group's detection algorithm that scales to networks with billions of edges, and these core groups are small cohesive groups of vertices that belong to the same community. These core groups' detection methods are pre processing methods for community detection [4].

\subsection{Community Detection methods with Edge Content in Social Media Networks.}

An algorithm was proposed for the purpose of detection of communities, which use the links between the nodes in order to determine the dense regions in the graph. The graph is actually the structure of social media networks and the dense regions are actually the communities of social media in the graph. The proposed method is basically based on the linkages structure in the social media network. So far, edge content in many recent applications is available, in order to provide better guidance in the process of detection of communities. 
Edges provide a much richer characterization of communities in social network, because the characteristics of pair wise interactions are modeled, rather than individual actors. The pair wise interaction content provides very specific information about the nature of the relationship between a particular pair of individuals and it implies the interactions of a single individual may be used in order to reflect their membership in different communities. The content on the edges in the representations of edges in social interactions can be shared images, videos, user tags and comments. The node content can be utilized for the detection of communities but the presence of edge content presents unprecedented opportunities and flexibility for the community detection process. Edge content can be leveraged for the improvement of the effectiveness of the community detection process in social media networks [2].

\subsection{Community Detection method with Influence Ranking in Social Networks}

The method for detecting communities along with influence analysis has been considered as significant concepts in social networks. The algorithm proposed for both community detection and influence ranking is purely based on the concept of influence-based connectivity and proximity encoded in the network topology. The proposed algorithm generates an influence vector for each node in the network using a new influence cascade model, which captures in detail how the node's influence is distributed through the network. In this algorithm the closeness of any pair of nodes is based on the similarity in the influence space which defines a new, meaningful and refined connectivity measure .The proposed method not only differentiates the influence ranking but also effectively finds communities in both undirected and directed networks, and incorporates these two important tasks into one integrated framework. This method was tested on a set of real world networks and also on synthetic networks. The procedure for the proposed method includes a new influence diffusion model that embeds influence into a node and passes it around in the network. The complexity of this algorithm in case of space is $O(n L)$, and for time it may take $O(b d)$, and time complexity in case of worst case is $O(n b d)$, where as $n$ denote the total number of nodes in the network, $b$ denote the average node out-degree, $d$ denote the depth limit, $K$ denote the number of communities, $I$ denote the number of iterations to converge, and $L$ denote the average length of the influence vectors [9].

\subsection{Community Detection method Using DBSCAN Algorithm in social network}

A community detection methods using DBSCAN algorithm was proposed, which is the most effective unsupervised clustering algorithm. From the graphical representation structure of social network, the interactions or connection between individuals or entities, or nodes can be viewed, from which the existence of communities can be concluded. In this approach detection of communities was done on the basis of three types of members in the community, which are core, border and outlier members, and which are of high, low and no influence respectively. The outliers were eliminated from the dataset because they are noises which are free to deal with it.The DBSCAN algorithm is robust to outliers, and by deleting the outliers the dataset will be noise free to deal with. The outliers can be detected by changing the radius (epsilon) of the cluster. In the analysis of social networks it mostly focuses on cores as they have influence to other members and the eliminated outlier member's leads to an accurate clustering result that helps with the community detection issue in the social network analysis field [10].

\subsection{Community Detection method by using Bayesian network and Expectation Maximization technique.}

It was observed that the communities in social networks have important roles in the structure function relationship. Communications between actors in the social networks are generated based on some assumptions and social networks can be formalized by a statistical model, so a statistical model was introduced which represents the interactions between social network's actors, and we use Bayesian network (probabilistic graphical model) to show the relation between model variables. A community detection algorithm based on the 
Expectation Maximization (EM) estimates was introduced, and is used to drive the estimates for the model parameters. The proposed approach works well with directed and undirected networks, and with weighted and un-weighted networks, and yields very promising results when applied to the community detection problem [11].

\subsection{Community detection method in a Social Network, using graph mining technique.}

It was observed that the extraction of knowledge is also possible from the community graph, using graph mining techniques. For detection of communities in a social network, graph mining techniques has been used. In this proposed approach, a community or a group is detected based on complete mutuality, nodes reachability and nodal degrees. In complete mutuality a group can be formed if a sub-graph is formed by considering more than two nodes and all are adjacent to each other which are termed as a clique. For reachability condition between two actors or nodes to be in a community, if there is a path between these two nodes. For nodal degrees it checks actors within a group to be adjacent to a relatively large number of group members or not [12].

\subsection{Community detection method based on spectral clustering.}

A new method is proposed to detect the number of communities based on spectral clustering. In this proposed method, the conductivity function and the accuracy are used to evaluate the quality of community detection. The bisection method based on the spectral clustering is one of the most common methods for community detection, based on Graph Spectral Theory. The proposed algorithm estimates the number of communities according to the eigen value distribution of the Laplacian matrix, and the k-means algorithm is applied for clustering. The proposed algorithm based on spectral clustering is applicable to the network graph which can be clearly divided into two communities, but actually the number of communities in common, cannot be detected [13]. A spectral clustering-based adaptive hybrid multi-objective harmony search algorithm (SCAH-MOHSA) combined with a local search strategy is proposed to detect the community structure in complex networks. In this approach, an improved spectral method is employed to convert the community detection problem into a data clustering issue while the length of the representation of a harmony in the harmony memory can be determined. After that, an adaptive hybrid multi-objective harmony search algorithm is used to solve the multi-objective optimization problem so as to resolve the community structure. The proposed algorithm is tested on both synthetic and real world networks, and demonstration of that method achieves partition results which fit the real situation in an even better fashion [14].

\subsection{Community detection method based on overlapping communities}

Along with community structure, overlap is also one of the attributes of social networks. These overlapping communities are detected when a person used to belong, to more than one social group or to more than one community. A person may belong to more than one community because usually he or she has connections to several social groups like friends, family institutions. Till now many overlapping communities have been proposed, which can be divide into two categories, node based and link based overlapping community detection algorithms. The node based overlapping community detection algorithms categorize the nodes of the network, and the link based overlapping community detection algorithms categorize the edges of network in clusters. For detection of overlapping communities researchers have proposed a parliamentary optimization algorithm which also helps to analyze to community structure [24].

\section{Some important observations in the existing approaches}

As we have already discussed some of the existing approaches. So in this section we are going to observe some advantages and disadvantage in the existing approaches. The first algorithm was proposed for the 
detection of communities in social network is Newman-Girvan algorithm which is used for detecting communities' structure in both computer generated and real world network data. [15]. For an algorithm was proposed for the detection of communities based on edge content and link content in the detection of communities in social media network. Edges provide a much richer characterization of community behavior, because the content models the characteristics of pair wise interactions rather than individual actors'.The authors have examined the proposed approach for email and flicker based approaches only [2]. One of the ways for the formation of communities is clustering, so the author's have proposed a Boolean programming problem for the partition of clusters. In this approach it ensures that only a fixed number of clusters should be selected and when a data object is required to be assign then a cluster must be selected. The proposed Boolean programming problem approach is not cleared by author's that for which type of network will it work appropriately[8].The proposed community detection scheme in an integrated internet of things (IoT) and Social Network (SN) architecture approach have chosen the graph mining approach for solving the problem present in complex network of IoT and SN. The approach for detection of communities in an integrated environment is very useful, because in the case of any search operation performed by any node, the results obtained for intracommunity are more relevant than intercommunity. This integrated environment approach fails to give results for directed networks, such as Twitter, and is not generalized to all networks [7]. An empirical study of community and sub-community detection reflects the structure of communities as well as sub-communities occurring in a social network by applying Newman-Girvan algorithm. The author's have implemented this community detection algorithm on real world networks. The results differ from those presented earlier in the sense that the author's have defined a new concept of sub-communities. The main drawback of NewmanGirvan algorithm is that the computational cost is relatively high [3]. The detection of communities in distributed environment in Web-scale networks based on an ensemble learning scheme which provides a way to identify high quality partitions from an ensemble of partitions with lower quality. Through the detection of communities in distributed environment it is possible to process a graph as large as $\sim 3.3$ billion edges on small Hadoop cluster with 50 nodes in just a few hours.[4]. Density based spatial clustering of applications with noise (DBSCAN), is also used in social network analysis. The DBSCAN algorithm has a notion of noise, and is robust to outliers. This approach is not an incremental method, which means it requires the whole data set in advance. Through this DBSCAN algorithm it is possible to eliminate outlier members which lead to an accurate clustering result that helps with the community detection issue in the social network analysis field [10]. Bayesian network (probabilistic graphical model) was used for showing the relationship between statistical model variables and statistical model is introduced for the interactions between social network's actors. The detection of communities was done based on the Expectation Maximization (EM) algorithm, which drive estimates for the model parameters. The algorithm works well for weighted, un-weighted networks and also for directed and un-directed networks [11]. Graph mining techniques are also very useful for extraction of knowledge from the community graph, so an algorithm based on graph mining techniques was proposed for the detection of communities. The graph mining technique approach is very much useful for large number of nodes and is examined for finding communities in villages [12].

\section{Tabulated view of different methods for detection of communities}


Table 1. Different methods with its advantages and disadvantages

\begin{tabular}{|c|c|c|c|}
\hline $\begin{array}{l}\text { SL. } \\
\text { No. }\end{array}$ & Algorithms & Advantages & Disadvantages \\
\hline 1. & $\begin{array}{l}\text { Community detection using } \\
\text { DBSCAN algorithm }\end{array}$ & $\begin{array}{l}\text { DBSCAN algorithm has a notion of } \\
\text { noise, and is robust to outliers. }\end{array}$ & $\begin{array}{l}\text { DBSCAN is not entirely } \\
\text { deterministic ,i.e. border } \\
\text { points that are reachable from } \\
\text { more than one cluster can be } \\
\text { part of either cluster }\end{array}$ \\
\hline 2. & $\begin{array}{l}\text { Community Detection method in } \\
\text { an Integrated Internet of Things } \\
\text { and Social Network Architecture }\end{array}$ & $\begin{array}{l}\text { An actor or a node in the network can be } \\
\text { part of multiple communities, similar to } \\
\text { real-life situation. } \\
\text { This approach can be used to suggest } \\
\text { friends }\end{array}$ & $\begin{array}{l}\text { This approach is not } \\
\text { generalized to all the } \\
\text { networks. It fails to give } \\
\text { results for directed } \\
\text { networks, such as twitter }\end{array}$ \\
\hline 3. & $\begin{array}{l}\text { Community Detection method } \\
\text { with Edge Content in Social } \\
\text { Media Networks. }\end{array}$ & $\begin{array}{l}\text { Edge content provides better supervision } \\
\text { to the community detection process }\end{array}$ & $\begin{array}{l}\text { This approach is useful for } \\
\text { email and flicker images } \\
\text { based clustering only. }\end{array}$ \\
\hline 4. & $\begin{array}{l}\text { Community Detection methods in } \\
\text { weighted network }\end{array}$ & $\begin{array}{l}\text { Total weight of all selected clusters in the } \\
\text { network is calculated and also the } \\
\text { similarity in between the clusters. }\end{array}$ & $\begin{array}{l}\text { The proposed approach is not } \\
\text { cleared for the type of } \\
\text { network, for it will work for. }\end{array}$ \\
\hline 5. & $\begin{array}{l}\text { Community \& sub -community } \\
\text { detection method using newman- } \\
\text { girvan algorithm }\end{array}$ & $\begin{array}{l}\text { Author's have defined a new concept of } \\
\text { detecting sub-communities }\end{array}$ & $\begin{array}{l}\text { Computational cost is } \\
\text { relatively high. }\end{array}$ \\
\hline 6. & $\begin{array}{l}\text { Community detection methods for } \\
\text { distributed environment, in Web- } \\
\text { Scale Networks. }\end{array}$ & $\begin{array}{l}\text { The proposed approach makes possible } \\
\text { to process a graph as large as } \sim 3.3 \\
\text { billion edges on small Hadoop cluster } \\
\text { with } 50 \text { nodes in just a few hours. }\end{array}$ & \\
\hline 7. & $\begin{array}{l}\text { Community Detection method by } \\
\text { using Bayesian network and } \\
\text { Expectation Maximization } \\
\text { technique. }\end{array}$ & $\begin{array}{l}\text { It works well with directed and } \\
\text { undirected networks, and with weighted } \\
\text { and un-weighted networks. }\end{array}$ & $\begin{array}{l}\text { It requires specifying the } \\
\text { number of communities in } \\
\text { advance. }\end{array}$ \\
\hline 8. & $\begin{array}{l}\text { Community detection method in a } \\
\text { Social Network, using graph } \\
\text { mining technique. }\end{array}$ & Useful for very large number of nodes. & \\
\hline 9. & $\begin{array}{l}\text { Community detection method } \\
\text { based on spectral clustering }\end{array}$ & $\begin{array}{l}\text { It not only find the real or near the real } \\
\text { partition, but also is able to find out } \\
\text { solutions with underling hierarchical } \\
\text { structures and fuzzy nodes that can } \\
\text { hardly be discovered by single objective } \\
\text { optimization approaches }\end{array}$ & \\
\hline 10. & $\begin{array}{l}\text { Community detection method } \\
\text { based on overlapping } \\
\text { communities. }\end{array}$ & $\begin{array}{l}\text { It detects the overlapping communities } \\
\text { and also analyze the community structure }\end{array}$ & $\begin{array}{l}\text { To detect the overlapping } \\
\text { community only modularity } \\
\text { method has been used as a } \\
\text { fitness function. }\end{array}$ \\
\hline
\end{tabular}




\section{Conclusion}

Communities play a significant role for the visualization of large social network which also helps in understanding the structure of social networks. This paper, describes different types of concepts and algorithms proposed, related to social network community detection. The introduction of social network community detection shows the different type of definitions for the social network community structure. Different type of algorithms i.e. the proposed approaches and their applications on the type of network for the community detection has also been discussed properly. The community detection significant applications have also been discussed .The observations of proposed approaches including the advantages and disadvantages have been shown through the table. The proposed approaches were mainly based on the mining algorithms and some of the graph mining approaches for the detection of communities in the social network. Thus in conclusion, I can state that all the proposed approaches that have been discussed will be useful for the other researcher's, doing research in the social network community detection.

\section{References}

[1] Mathilde Forestier, Anna Stavrianou, Julien Velcin, and Djamel A. Zighed, "Roles in social networks: methodologies and research issues", Web intelligence and Agent Systems: An international Journal 10 (2012) 117-133.

[2] Guo-Jun Qi1, Charu C. Aggarwal2 and Thomas Huang1, "Community Detection with Edge Content in Social Media Networks", Data Engineering (ICDE), 2012 IEEE 28th International Conference.

[3] Deepjyoti Choudhury, Saprativa Bhattacharjee, Anirban Das, "An Empirical Study of Community and Sub-Community Detection in Social Networks Applying Newman-Girvan Algorithm”, Emerging Trends and Applications in Computer Science (ICETACS), 2013 1st International Conference.

[4] Michael Ovelgonne, "Distributed Community Detection in Web-Scale Networks", Advances in Social Networks Analysis and Mining (ASONAM), 2013 IEEE/ACM International Conference.

[5] Mary McGlohon, Leman Akoglu and Christos Faloutsos, "Statiscal properties of social networks," C. C. Aggarwal (ed.), Social Network Data Analytics, Springer Science + Business Media, LLC 2011.

[6] Andry Alamsyah, Budi Rahardj, Kuspriyanto, "Community Detection Methods in Social Network Analysis", 2011 American Scientific Publishers.

[7] Sudip Misra, Romil Barthwal, Mohammad S. Obaidat, "Community Detection in an Integrated Internet of Things and Social Network Architecture", Global Communications Conference (GLOBECOM), 2012 IEEE.

[8] Ganjaliyev. F, "New Method for Community Detection in Social Networks Extracted from the Web", Problems of Cybernetics and Informatics (PCI), 2012 IV International Conference, IEEE.

[9] Wenjun Wang and W. Nick Street, "A Novel Algorithm for Community Detection and Influence Ranking in Social Networks", 2014 IEEE/ACM International Conference on Advances in Social Networks Analysis and Mining (ASONAM 2014).

[10] Yomna M. ElBarawy, Ramadan F. Mohamedt and Neveen I. Ghali, "Improving Social Network Community Detection Using DBSCAN Algorithm", Computer Applications \& Research (WSCAR), 2014 World Symposium, 2014 IEEE.

[11] Ahmed Ibrahem Hafez, Abaul ella Hassanien, Aly A. Fahm and M.F.Talba, "Community Detection in Social Networks by using Bayesian network and Expectation Maximization technique", 2013 IEEE.

[12] Bapuji Rao, Anirban Mitra , "A New Approach for Detection of Common Communities in a Social Network using Graph Mining Techniques", 2014 IEEE. 
[13] Jing Qiu, Jing Peng Ying Zhai, "Network community detection based on spectral clustering", Proceedings of the 2014 International Conference on Machine Learning and Cybernetics, Lanzhou, 1316 July, 2014.

[14] Yangyang Li, Ruochen Liu and Jianshe Wu, "A Spectral Clustering-Based Adaptive Hybrid MultiObjective Harmony Search Algorithm for Community Detection”, WCCI 2012 IEEE World Congress on Computational Intelligence June, 10-15, 2012 - Brisbane, Australia.

[15] M. E. J. Newman, M. Girvan, "Finding and evaluating community structure in networks", Phys. Rev. E, Columbia, vol. 69, pp. 1-15, August 2003.

[16] Maryam pourkazemi, Mohammadreza Keyvanpour, "A survey on community detection methods based on the nature of social networks", 3rd International Conference on Computer and Knowledge Engineering (ICCKE 2013), October 31 \& November 1, 2013, Ferdowsi University of Mashhad.

[17] Social Network Analysis Theory and Applications, http://train.ed.psu.edu/WFED543/SocNet_TheoryApp.pdf.

[18] Yuan Huang, Wei Hou, Xiaowei Li and Shaomei Li, "An Effective Community Detection Algorithm of the Social Networks", Third International Conference on Information Science and Technology March 23-25, 2013; Yangzhou, Jiangsu, China.

[19] Dr. S. Vijayarani1, Ms. P. Jothi, "An Efficient Clustering Algorithm for Outlier Detection in Data Streams" , International Journal of Advanced Research in Computer and Communication Engineering Vol. 2, Issue 9, September 2013.

[20] Hitesh R.Gor, Maulik V. Dhamecha, "A Survey on Community Detection in Weighted Social Network", International Journal of Advance Research in Computer Science and Management Studies, Volume 2, Issue 1, January 2014.

[21] Karsten Steinhaeuser1 and Nitesh V. Chawla, "Community Detection in a Large Real-World Social Network", http://www-users.cs.umn.edu/ ksteinha/papers/SBP08.pdf.

[22] Deng Cai, Zheng Shao, Xiaofei He, Xifeng Yan, Jiawei Han, "Mining Hidden Community in Heterogeneous Social Networks", Proceedings of the $3^{\text {rd }}$ international workshop on Link Discovery Pages 58 - 65, ACM New , York, NY, USA ,2005.

[23] Mahadevan Vasudevan and Narsingh Deo, "Efficient community identification in complex networks", 11 July 2012, Springer-Verlag 2012.

[24] Feyza Altunbey and Bilal Alatas, "Overlapping Community Detection in Social Networks Using Parliamentary Optimization Algorithm”, International Journal of Computer Networks and Applications Volume 2, Issue 1, January - February (2015).

\section{Author's profile}

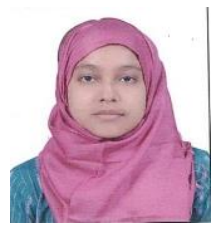

Mehjabin Khatoon (born $1^{\text {st }}$ July 1988) is a research scholar in B. S. Abdur Rahman University, Chennai, India. She did her B.Tech in Computer science \& engineering from Galgotias College of Engg. \& Technology ,Greater Noida ,Uttar Pradesh in the year 2009, and her M.Tech from JSS College of Academy and Technical Education, Noida, Uttar Pradesh in the year 2012. Her research focus is mainly in the area of Social Web Mining.

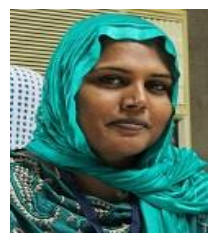

Dr. W.Aisha Banu: Her research focus is primarily in the area of Information Retrieval and Natural language processing. She has a rich teaching experience of seventeen years. She has published research papers in peer reviewed journal and conferences. She is a life member of ISTE and member of ACM 